\title{
Optimal wind farm cable routing: Modeling branches and offshore transformer
} modules

Fischetti, Martina; Pisinger, David

Published in:

Networks

Link to article, DOI:

10.1002/net.21804

Publication date:

2018

Document Version

Peer reviewed version

Link back to DTU Orbit

Citation (APA):

Fischetti, M., \& Pisinger, D. (2018). Optimal wind farm cable routing: Modeling branches and offshore transformer modules. Networks, 72(1), 42-59. https://doi.org/10.1002/net.21804

\section{General rights}

Copyright and moral rights for the publications made accessible in the public portal are retained by the authors and/or other copyright owners and it is a condition of accessing publications that users recognise and abide by the legal requirements associated with these rights.

- Users may download and print one copy of any publication from the public portal for the purpose of private study or research.

- You may not further distribute the material or use it for any profit-making activity or commercial gain

- You may freely distribute the URL identifying the publication in the public portal

If you believe that this document breaches copyright please contact us providing details, and we will remove access to the work immediately and investigate your claim. 


\title{
Optimal wind farm cable routing: modeling branches and offshore transformer modules
}

\author{
Martina Fischetti ${ }^{1,2}$ \\ Vattenfall BA Wind and \\ Technical University of Denmark, \\ DTU Management Engineering, Operations Research, \\ Produktionstorvet, 424, DK-2800 Kgs. Lyngby, Denmark \\ David Pisinger ${ }^{3}$ \\ Technical University of Denmark, \\ DTU Management Engineering, Operations Research, \\ Produktionstorvet, 424, DK-2800 Kgs. Lyngby, Denmark
}

\begin{abstract}
Many EU countries aim at reducing fossil fuels in the near future, hence an efficient production of green energy is very important to reach this goal. In this paper we address the optimization of cable connections between turbines in an offshore wind park. Different versions of the problem have been studied in the recent literature. As turbines are becoming still more customized, it is important to be able to evaluate the impact of new technologies with a flexible optimization tool for scenario evaluation. In a previous joint project with Vattenfall BA Wind (a global leader in energy production) we have studied and modelled the main constraints arising in practical cases. Building on that model, in the present paper, we address new technological features that have been recently proposed by Vattenfall's experts. We show how some new features can be modelled and solved using a Mixed-Integer Linear Programming paradigm. We report and discuss computational results on
\end{abstract}


the performance of our new models on a set of real-world instances provided by Vattenfall.

Keywords: Network models, wind farm optimization, mixed-integer linear programming, computational analysis.

\section{Introduction}

The production of green (in particular, wind) energy is an important instrument in limiting the climate changes of the world. As modern wind parks are getting bigger in size and in produced power, it is very important to optimize their design. Designing a wind farm is, however, a complex process including selection of the right site, optimizing the location of each turbine $[11,14]$, establishing the infrastructure [2] and connecting the farm to the existing electrical grid [22].

In this paper we address the optimization of cable connections among offshore turbines, called cable routing in what follows. When turbines are located offshore their energy production is first transmitted to one or more collection points (substations) using lower voltage cables (33 or $66 \mathrm{kV}$ ), called inter-array cables. The energy is then moved from the substations to shore using higher voltage cables, called export cables. We will here focus on the inter-array cable optimization. The original version of this problem consists of connecting all the offshore turbines to one (or more) offshore substation(s), minimizing the total cable cost. The final cable layout has a tree structure where the non-root nodes correspond to the given turbines, the substations play the role of roots, and the energy (i.e., the electric current) flows from the nodes to the roots along the tree. Figure 1 gives an example of cable layout for a real wind park.

A number of constraints must be taken into account when designing a feasible cable routing. First of all, the energy flow is unsplittable, i.e., the flow leaving a turbine must be supported by a single cable. In addition, each substation has a physical layout that imposes a maximum number of entering cables. Cable crossings should be avoided, as establishing one cable across

$\overline{1}$ Work supported by Innovation Fund Denmark. Thanks to Jesper Runge Kristoffersen, Iulian Vranceanu, Thomas Hjort, Mads Krogsgaard and Kenneth Skaug from Vattenfall BA Wind who helped us in defining the cable routing constraints.

2 Email: martina.fischetti@vattenfall.com

3 Email: dapi@dtu.dk 


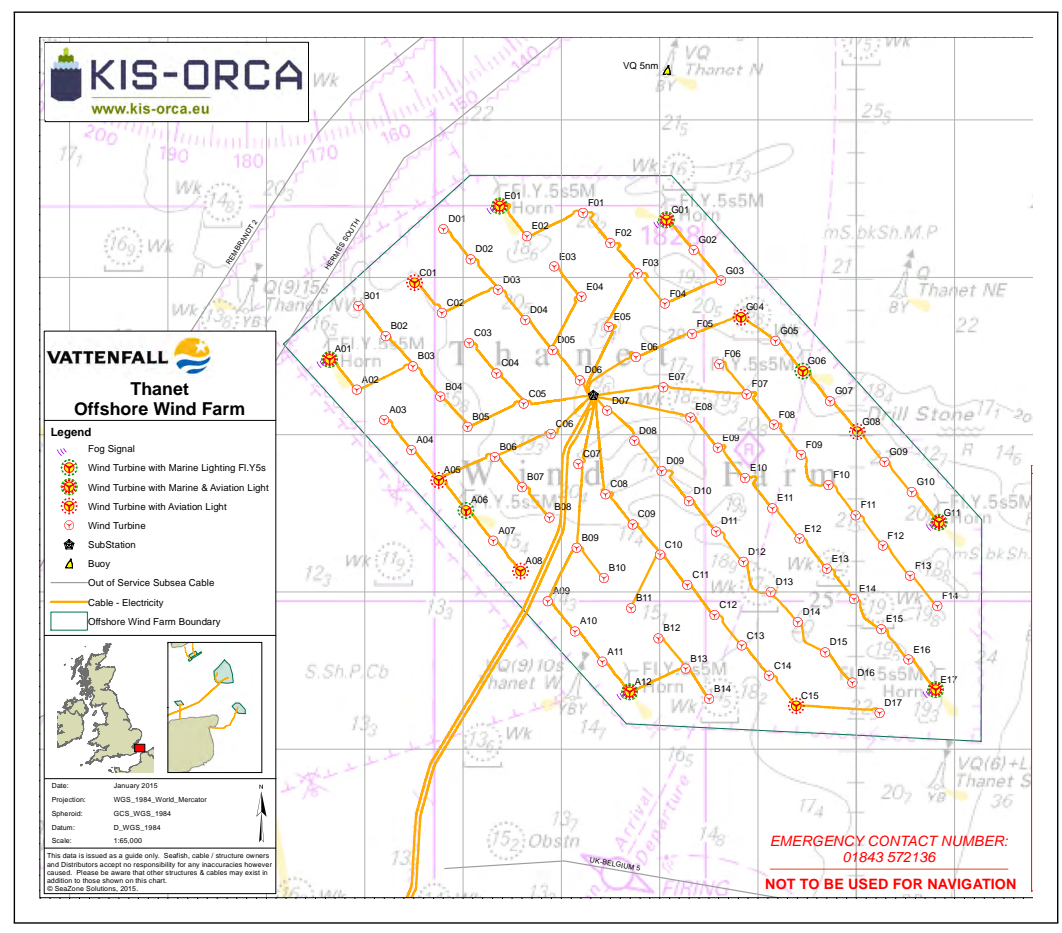

Fig. 1. An example of cable routing for a real-world offshore wind park (Thanet) owned by Vattenfall-picture from [18].

another is expensive and increases the risk of cable damages.

Several types of cables with different costs and capacities are available on the market. Therefore, one has to also optimize the cable type selection in order to deliver all the energy production to the substations at minimum cost. In our collaboration with Vattenfall, we had the chance to have a close look at how engineers are evaluating different scenarios and technological possibilities, to design competitive wind parks. Earlier, most of this work was carried out manually, so evaluating different possibilities was very difficult and time consuming. We closely collaborated with different engineer teams in Vattenfall, to model and optimize different versions of the cable routing problem arising in practice. This family of problems has received limited attention in the OR community so far, so we aim here at describing and modelling some new optimization problems from an OR perspective, while also showing the impact of having sound optimizers to help engineers in practice. To be more specific, we will first describe different versions of the classical cable routing problem arising in practical applications, and then we will compare the resulting layouts following the "what-if" analysis approach that is carried out by the company before selecting one technology instead of another. 
The basic formulation of the wind park cable routing optimization problem has received significant attention in the OR literature in the last years. Due to the large number of constraints and the intrinsic complexity of the problem, many papers (including $[8,15,20,21,25]$ among others) prefer to use ad-hoc heuristics. Just few articles from the literature use Mixed Integer Linear Programming (MILP) for cable routing; see e.g. [2,3,6,7,9,17].

Compared to Bauer's definition [2] of the Offshore Wind Farm Array Cable Layout (OWFACL) problem, our initial formulation also includes substation limitations and the possibility of having different cable types. Therefore, we decided to introduce a new name for our version of the problem, denoted the Offshore Wind Farm Cable Routing (OWFCR) problem. The new formulation allows for multiple substations as well.

The basic formulation of OWFCR has been studied by the present authors in [12], considering also additional technical features such as obstacles in the site and power losses in the cables.

Due to our ongoing collaboration with Vattenfall, we have a continuous feedback from experts on the new problem specifications arising from upcoming projects. Wind energy is a highly-competitive and a relatively new field, where technology is still quickly developing. Suitable MILP models that can capture new technology requirements are therefore very valuable in scenario evaluation as a substitute for, or a complement to, the existing manual design process. In this paper we will therefore look at different possible scenarios for the cable routing problem, where different technological requirements are considered. We will see how the basic MILP model (OWFCR) can be extended to evaluate these scenarios and be used to quantify their impact on the design of offshore wind parks. In particular, we will present four different extensions of the original OWFCR model, that will be discussed in details in the next sections.

The paper is organized as follows. In Section 2 we recall the original version of the cable routing problem (OWFCR) and explain how to model its basic constraints using Mixed Integer Linear Programming. When discussing the resulting layouts with practitioners, we received different additional requirements that we included in the new versions of the model.

First, we considered an engineering requirement on the topology of the route: we were asked to evaluate the impact of connecting turbines only in strings, i.e., to have at most one cable entering and exiting each turbine. The resulting model will be denoted as OWFCR-SS (String Structure), and will be studied in Section 3. Secondly we were asked to not impose any specific topology, but explicitly consider the possible additional costs for connecting 
more cables to each turbine (as additional switch-gears may be needed in some turbine models). This version of the model is denoted OWFCR-BP (as it considers Branching Penalties). It is studied in Section 4.

Even though we could show that a layout with branches is less expensive from an immediate cost perspective, it could be difficult for the company to detect cable failures in such a structure. Therefore, the company proposed an additional scenario, where turbines must be connected in strings and the strings must be coupled in loops to create fault-tolerant cycles. The problem of constructing fault-tolerant networks is well know in the telecommunication literature, where different topologies have been studied (see, e.g. $[5,13,19]$ ). Nevertheless, in the considered wind farm application, we were asked by the company to consider a very specific topology, namely, a so-called closed-loop structure. Since the aim of our work is to answer the practical needs of our industrial partner, we decided to stick to this structure. A closed-loop (or ring) structure is characterized by the presence of redundant minimum-capacity cables between strings, that avoid disconnected turbines in case of cable failure. We analyze this structure in Section 5, where we extend our model to handle ring structures, resulting in our third variant of the model (OWFCR-CL). As we will see also in our computational section, this is a more expensive structure (compared with the original branch structure) so it should be used only if necessary (i.e., if turbines are not equipped to survive cable failures by other means).

In Section 6 we consider the extension of the OWFCR model where there is no offshore substation, but a smaller Offshore Transformer Module (OTM) can be installed on normal turbines. The turbines equipped with this OTM can be connected to both inter-array cables (small cables that connect turbines one with each other) and to the export cable (higher voltage cable, that connects turbines to shore). The new variant of the problem, named OWFCR-OTM, is a particularly interesting problem variant, since it involves also the decision of how many OTMs should be installed and on which turbines. Given the high cost of offshore substations, a properly optimized layout with OTMs can greatly reduce the overall costs. Each of the new models extends the original OWFCR model, as shown in the diagram in Figure 2.

Due to the complexity and size of the studied problems, we used matheuristic techniques to speed-up the solution of the previously described models. Section 7 briefly describes the hybrid matheuristic/exact algorithm we used. Section 8 reports our tests of the new models on a set of real-world instances, 


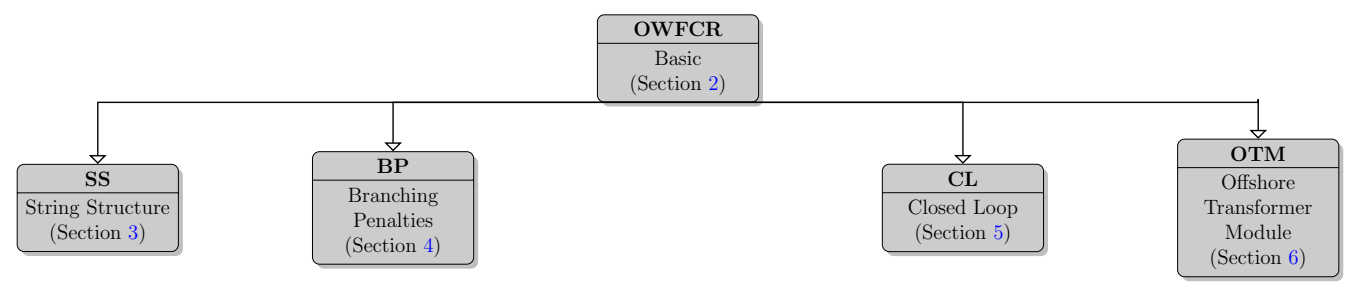

Fig. 2. Taxonomy of the OWFCR problem variants

while some conclusions are drawn in Section 9.

\section{The basic MILP model (OWFCR)}

We first need to briefly recall the basic model (OWFCR) we developed for Vattenfall; the reader is refereed to [12] for further details.

Assuming that turbine positions are fixed and a set of cable options (with different capacities and costs) is given, the OWFCR problem is to find an optimal cable connection between all turbines and the given substation(s), minimizing the total cable cost. The network must ensure that the energy flow on each link does not exceed the capacity of the installed cable, and the energy flow leaving a turbine is supported by a single cable. An additional technical requirement is that a given maximum number of cables, say $C$, can be connected to each substation. Finally, cable crossings should be avoided to reduce the risk of damages.

\subsection{Mathematical formulation}

Turbines can be represented by nodes in a complete and loop-free directed graph $G=(V, A)$, and all possible connections between them by directed arcs. Some nodes correspond to the substations that are considered as the roots of the distribution network, and are the only nodes that collect energy. The final solution consists of a set of trees rooted at the substations whose arcs are directed from the nodes to the roots, following the energy flow. The model also allows for optional "Steiner" nodes, that can either be left uncovered, or have exactly one entering and one leaving cable. These dummy nodes are useful when considering obstacles in the area, or to allow for curvy connections between two nodes; see [12] for details.

Each node corresponds to a point in the plane, whose coordinates are used to compute distances between nodes as well as to determine whether two given line segments $[i, j]$ and $[h, k]$ cross each other, where $[a, b]$ denotes the line segment in the plane having nodes $a, b \in V$ as endpoints. It is assumed 
that two line segments meeting at one extreme point do not cross each other. Analogously, two segments do not cross if one is contained in the other, as they correspond to two parallel cables that can be physically built one besides the other without crossing issues.

The node set $V$ is partitioned into $\left(V_{T}, V_{0}, V_{S}\right)$, where $V_{T}$ contains the nodes corresponding to the turbines, $V_{0}$ contains the nodes corresponding to the substations, and $V_{S}$ contains the Steiner nodes (if any). Furthermore, let $P_{h} \geq 0$ denote the power production at node $h \in V$, where $P_{h}>0$ for $h \in V_{T}$ and $P_{h}=0$ for $h \in V_{S}$ (nodes $h \in V_{0}$ corresponding to substations have $P_{h}=-1$ by convention).

Let $T$ denote the set of different cable types that can be used. Each cable type $t \in T$ has a given capacity $k_{t} \geq 0$ and a unit cost $u_{t} \geq 0$. Arc costs $c_{i, j}^{t}=u_{t} \cdot \operatorname{dist}(i, j)$ can then be computed for each arc $(i, j) \in A$ and for each cable type $t \in T$, where $\operatorname{dist}(i, j)$ is the Euclidean distance between nodes $i$ and $j$.

Decision variables are as follows. For each $\operatorname{arc}(i, j) \in A$, we have a continuous variable $f_{i, j} \geq 0$ representing the (directed) energy flow from $i$ to $j$, and a binary variable $x_{i, j}^{t}=1$ iff arc $(i, j)$ is constructed with cable type $t \in T$. Finally, binary variables $y_{i, j}=\sum_{t \in T} x_{i j}^{t}$ indicate whether an $\operatorname{arc}(i, j)$ is built with any type of cable.

The MILP model presented in [12] will be denoted OWFCR, and it is defined by:

$$
\begin{aligned}
\min \sum_{(i, j) \in A} \sum_{t \in T} c_{i, j}^{t} x_{i, j}^{t} & \\
\sum_{t \in T} x_{i, j}^{t} & =y_{i, j}, \quad(i, j) \in A \\
\sum_{i \in V: i \neq h}\left(f_{h, i}-f_{i, h}\right) & =P_{h}, \quad h \in V_{T} \cup V_{S} \\
\sum_{t \in T} k_{t} x_{i, j}^{t} & \geq f_{i, j}, \quad(i, j) \in A \\
\sum_{j \in V: j \neq h} y_{h, j} & =1, \quad h \in V_{T} \\
\sum_{j \in V: j \neq h} y_{h, j} & =0, \quad h \in V_{0} \\
\sum_{j \in V: j \neq h} y_{h, j} & \leq 1, \quad h \in V_{S}
\end{aligned}
$$




$$
\begin{aligned}
\sum_{i \in V: i \neq h} y_{i, h} & \leq 1, \quad h \in V_{S} \\
\sum_{i \in V: i \neq h} y_{i, h} & \leq C, \quad h \in V_{0} \\
y_{i, j}+y_{j, i}+y_{h, k}+y_{k, h} & \leq 1, \quad \text { for all crossing segments }[i, j] \text { and }[h, k] \\
x_{i, j}^{t} & \in\{0,1\},(i, j) \in A, t \in T \\
y_{i, j} & \in\{0,1\},(i, j) \in A \\
f_{i, j} & \geq 0, \quad(i, j) \in A .
\end{aligned}
$$

The objective function (1) minimizes the total cable layout cost. Constraints (2) say that only one type of cable can be selected for each built arc, and define the $y_{i, j}$ variables. Constraints (3) stipulate that the energy (flow) exiting each node $h$ is equal to the energy entering $h$ plus the power production of that node; these constraints are not imposed for $h \in V_{0}$, i.e., when $h$ corresponds to a substation. Constraints (4) instead ensure that the flow does not exceed the capacity of the installed cable. Constraints (5) impose that only one cable leaves a turbine, whereas constraints (6) say that no cable can exit a substation, thus enforcing a tree structure rooted at the substation(s). As to Steiner nodes, it is optional to connect them but, if they are connected, only one cable can enter these nodes (constraints (7)). Furthermore, (8) imposes that at most one cable can exit a Steiner node. Constraint (9) imposes the maximum number of cables $(C)$ that can enter each substation. Finally, inequalities (10) forbid building any two crossing arcs.

No-cross constraints (10) can be strengthened by exploiting constraints (5)(7), so as to reduce their number and to improve their quality. To this end, for any node triple $(a, b, k)$, let the "clique" arc subset $\mathcal{Q}(a, b, f)$ be defined

$$
\mathcal{Q}(a, b, f)=\{(a, b),(b, a)\} \cup\{(f, h) \in A \text { : segments }[a, b] \text { and }[f, h] \text { cross }\}
$$

The following improved no-cross constraints have been shown to be valid in [12]:

$$
\sum_{(i, j) \in \mathcal{Q}(a, b, f)} y_{i, j} \leq 1, \quad a, b, f \in V, a \neq b, b \neq f, f \neq a .
$$

\subsection{Example}

First we will illustrate the result of our OWFCR model with a real-world example. We consider the Horns Rev 3 (HR3) case, a 350 MW park in Denmark, still under construction. Fifty $8 \mathrm{MW}$ turbines are used in the layout (they are represented as black dots in the figure plots). In this park, the offshore 
substation is given by the grid operator, thus its position is fixed. At most 12 cables can be connected to the substation (red square in Figures 3). Our set of cables consists of three types of cable: the black one supports 3 turbines at a cost of $393 € / \mathrm{m}$, the green one supports 4 turbines at a cost of $460 € / \mathrm{m}$, and the blue one supports 5 turbines at a cost of $540 € / \mathrm{m}$ (costs include both cable and installation costs). HR3 will be used as an example park also for the OWFCR model variants, in the next sections. A further comparison between the different models on various real-world wind parks is presented in Section 8.

The OWFCR model results in the optimized layout of Figure 3 and does not consider other additional costs or constraints. This optimal solution was found in 176 seconds on a standard PC, using the hybrid matheuristic/exact framework of Section 7.

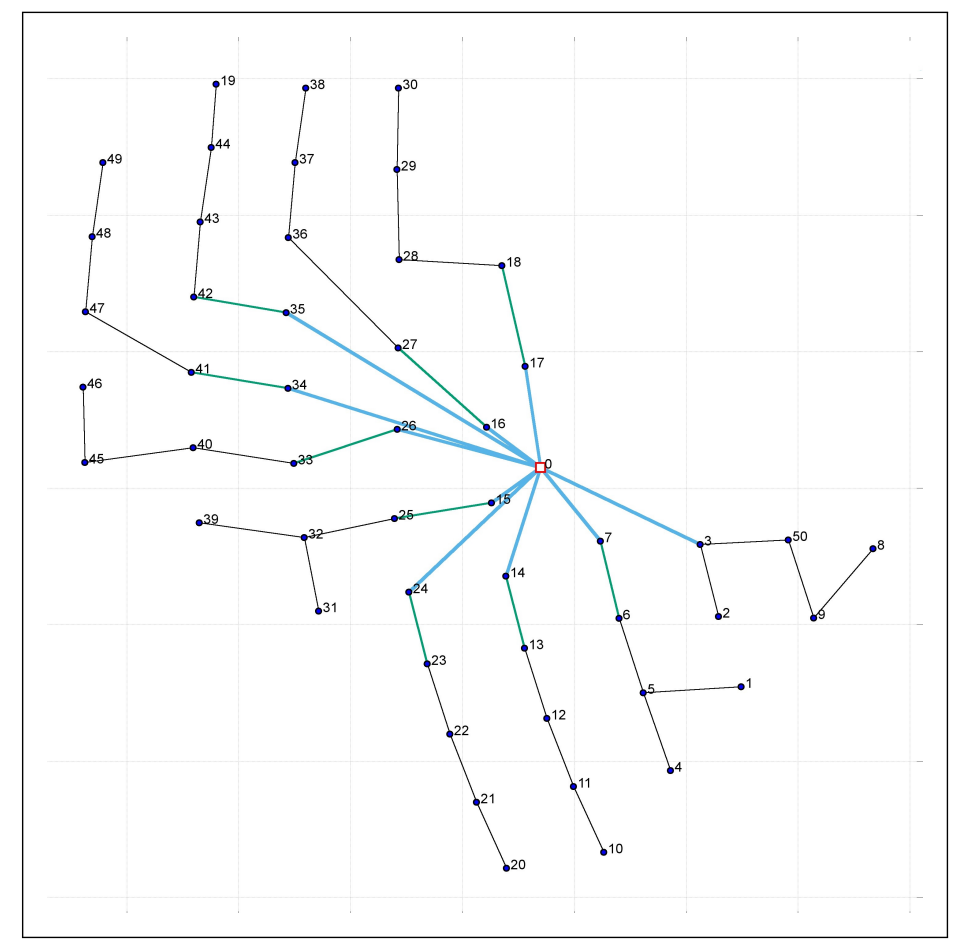

Fig. 3. Optimal solution of the the OWFCR problem for HR3. Power is transmitted from the leaves towards the root (node 0 , marked with a red square).

\section{String structure (OWFCR-SS)}

The OWFCR model presented in Section 2 often constructs cable routings with 
branches, as seen in the HR3 example in Figure 3. Branches are structures with more than two cables entering a turbine (as node 3, 5, and 32 in Figure $3)$. Branches are not impossible in practice but they can involve extra costs for the additional hardware (load breaker or disconnectors). This depends on the turbines used: some of them are equipped with hardware allowing for multiple connections by default, some of them do not. For turbines with multiple connections the OWFCR problem formulation holds, while for turbines with only one entering cable connection, we were ask to evaluate a scenario where the string structure is enforced. This additional requirement gives rise to the OWFCR-SS problem variant.

\subsection{Mathematical formulation}

The OWFCR model is extended with the following constraints:

(15) $\sum_{i \in V: i \neq h} y_{i, h} \leq 1, h \in V_{T}$,

imposing that at most one cable can enter each turbine. The OWFCR-SS model is then the OWFCR model (1)-(14) with the addition of constraint (15).

\subsection{Example}

As an example of an optimized layout imposing a string structure we again use the HR3 case. We run the OWFCR-SS model on a standard PC with a time limit of 1 hour. The resulting layout is shown in Figure 4, and it is 222 $\mathrm{k} €$ more expensive than the one in Figure 3. This optimal solution was found in 598 seconds on a standard PC, using the approach described in Section 7.

\section{Branching Penalties (OWFCR-BP)}

Having quantified the cost impact of imposing a string structure on the layout, the company was interested in finding a middle-way solution. Knowing the cost of the additional hardware for branches, and having a tool able to consider this in the optimization, the company would like to find the the optimal indegree for each turbine in a layout. Note the the extra costs (called branch penalties in what follows) depend on the number of arcs entering each turbine, but not in a linear way. We therefore studied another version of the original OWFCR problem, explicitly including branching penalties in the optimization (denoted as the OWFCR-BP). 


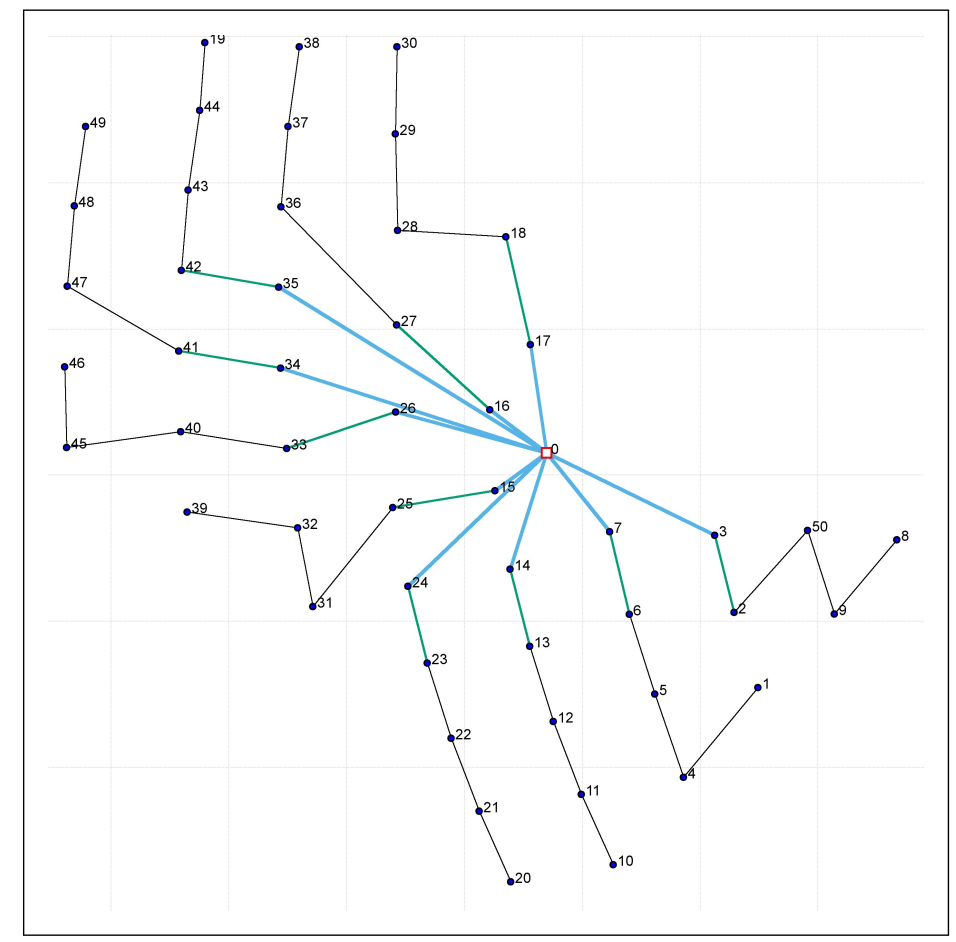

Fig. 4. Imposing a string structure, i.e., solution of the OWFCR-SS problem.

\subsection{Mathematical formulation}

We describe the modifications needed to deal with branch penalties in the OWFCR model. Let $d_{\max }$ be the maximum allowed in-degree for a node (in typical applications, $d_{\max }$ is 2 or 3$)$, and let $D=\left\{1, \cdots, d_{\max }\right\}$. Moreover, for each possible number of entering cables $d \in D$, let $\pi_{d}$ be the extra-cost (penalty) incurred for each node in $V_{T}$ that has in-degree equal to $d$ in the final solution. In our study we considered turbines with at most two entering cables. The standard technology for turbines includes connections for only one entering and leaving cable (this configuration has zero extra cost, so $\pi_{1}=0$ ). If we want to have more than one entering cable (branch structure), we have to pay for the additional load breakers or disconnectors, and for the extra time to install them. Referring to our test-case HR3, Vattenfall's experts estimated that the extra cost for having two cables entering a turbine is $15 \mathrm{k} €$ for the disconnector, plus $10 \mathrm{k} €$ for installation (therefore, $\pi_{2}=25 \mathrm{k} €$ ).

We introduce a new set of binary variables $z_{j}^{d}$ with $j \in V_{T}$ and $d \in D$, where $z_{j}^{d}=1$ iff the in-degree of node $j$ is equal to $d$. The objective function for the OWFCR-BP then reads 


$$
\min \sum_{(i, j) \in A} \sum_{t \in T} c_{i, j}^{t} x_{i, j}^{t}+\sum_{d \in D} \pi_{d} \sum_{j \in V_{T}} z_{j}^{d}
$$

while we add the following additional constraints:

$$
\begin{gathered}
\sum_{i \in V: i \neq j} y_{i, j}=\sum_{d \in D} d z_{j}^{d}, \quad j \in V_{T} \\
\sum_{d \in D} z_{j}^{d} \leq 1, \quad j \in V_{T} \\
z_{j}^{d} \quad \in\{0,1\}, \quad j \in V_{T} .
\end{gathered}
$$

Model OWFCR-BP is therefore the OWFCR model (2)-(14) with the addition of constraints (17)-(19), and with (16) replacing the objective function.

Note that, in (18), we write " $\leq$ " instead of "=" so as to allow for leaf nodes with zero in-degree.

\subsection{Example}

Figure 5 plots the layout obtained by considering the HR3 case with the same constraints as in the OWFCR model, but imposing that at most two cables can enter a turbine and that the extra cost for each of these branches is 25 $\mathrm{k} €$. This optimal solution was found in 232 seconds on a standard PC. The structure of the solution is not really affected by the extra costs, and only slightly changes from Figure 3, nevertheless this solution is $20 \mathrm{k} €$ cheaper than the one in Figure 3 and about $200 \mathrm{k} €$ cheaper than the one in Figure 4.

\section{Closed-loop structure (OWFCR-CL)}

A main reason to consider the so-called closed-loop structure is to cope with cable failures. Generally speaking, turbines are designed to be connected to an electrical grid. Modern turbines are manufactured to have a certain autonomy to survive disconnection from the grid, but less recent models do not have this feature. This means that, in case of cable failures, the disconnected turbines could suffer from major damages. In order to avoid this situation, parks with this kind of turbines need to use redundant cables (or expensive batteries/diesel generators attached to each turbine). The main purpose of these redundant cables is to keep all turbines connected to the grid, in case an inter-array cable failure occurs. Note that this extra cable does not need to transport all the produced power to the substation, since turbines are curtailed to reduce their power production in case of cable failure. A specific redundant cable-routing structure was required by our company partner, that we call 


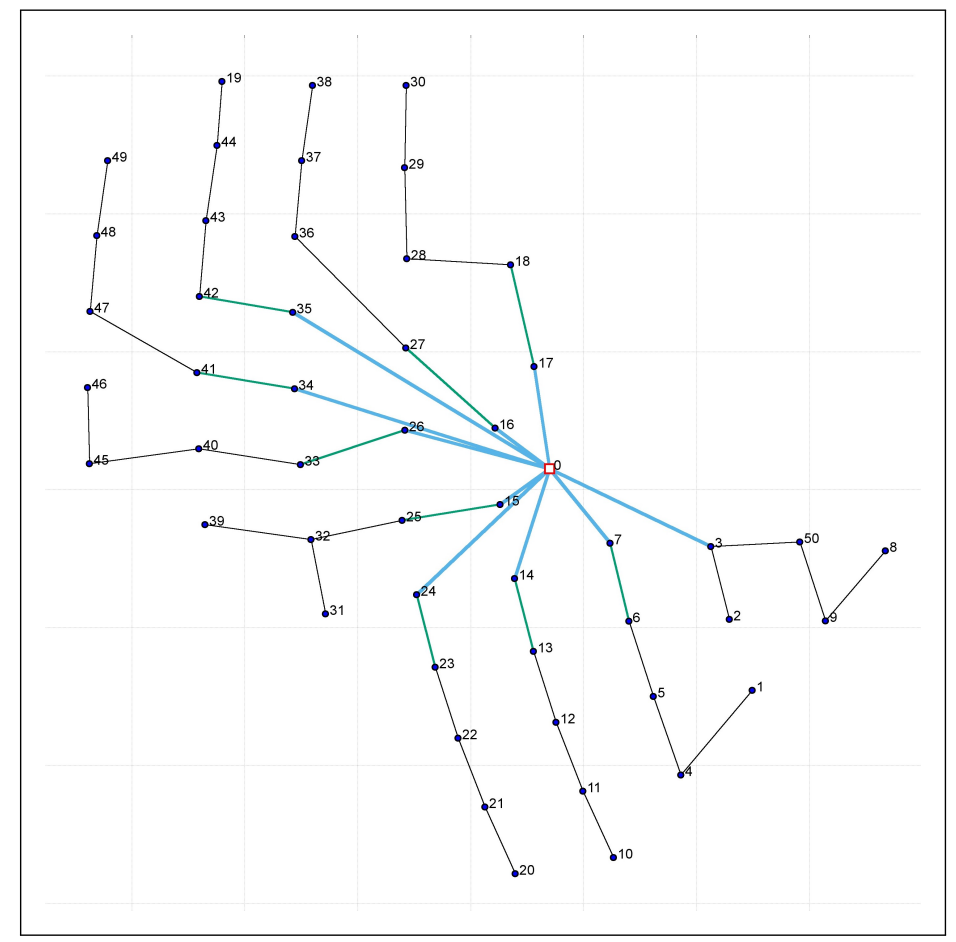

Fig. 5. Including branch penalties: solution of the OWFCR-BP problem.

closed-loop (or ring) structure. This is the redundant structure most used in practice, because it has high reliability and permits ease of fault location [23]. This structure consists in having at most one cable entering a turbine (as in the OWFCR-SS formulation) and in pairing the leaf turbines by redundant cable connections; see Figure 6 for an illustration. These connections always use only the cheapest cable available, because they are only intended to keep the turbines connected to the grid in case of a cable failure. This new variant of the OWFCR problem with closed loops, will be denoted by OWFCR-CL.

\subsection{Mathematical formulation}

To impose the closed-loop structure in the OWFCR model we introduce a new binary variable $q_{i, j}$ for each $(i, j) \in A$, where $q_{i, j}=1$ if a redundant cable has to be installed between nodes $i$ and $j$. These variables are added to the original OWFCR model, so that the new model will find a min-cost set of rings. As the redundant cable connections have no orientation, we actually fix $q_{i, j}=0$ whenever $i>j$, thus halving the number of additional variables required. 
The new variables $q_{i, j}$ are then linked to the $y_{i, j}$ through the following constraints to be added to the basic OWFCR MILP model:

$$
\begin{array}{cc}
\sum_{i \in V: i \neq h}\left(y_{i, h}+y_{h, i}+q_{i, h}+q_{h, i}\right)=2 \sum_{j \in V: j \neq h} y_{h, j}, & h \in V_{T} \cup V_{S} \\
q_{i, j}=0, & (i, j) \in A: i>j \\
q_{i, j} \in\{0,1\}, & (i, j) \in A .
\end{array}
$$

Note that the degree-2 constraints (20) automatically impose a string structure, with an even number of strings paired into rings. These constraints are not imposed for nodes $h \in V_{0}$ that correspond to substations (that are allowed to have degree 4 or more), and that the right-hand side term is zero in case node $h \in V_{S}$ is left uncovered. To avoid that the new arcs induce crossings in the final routing, in our branch-and-cut solver we dynamically separate (for integer solutions only) the following extended no-cross constraints

(23) $y_{i, j}+y_{j, i}+y_{h, k}+y_{k, h}+q_{i, j}+q_{j, i}+q_{h, k}+q_{k, h} \leq 1$

for each pair $[i, j]$ and $[h, k]$ of crossing edges.

As to the objective function, each new variable $q_{i, j}$ has a cost computed as $c_{i, j}^{t_{\text {min }}}=u_{\text {min }} \cdot \operatorname{dist}(i, j)$, where $u_{\text {min }}=\min _{t \in T} u_{t}$ is the unit cost of the least expensive cable. These costs are added to the OWFCR objective function (1) to obtain:

(24) $\min \sum_{(i, j) \in A} \sum_{t \in T} c_{i, j}^{t} x_{i, j}^{t}+\sum_{(i, j) \in A} c_{i, j}^{t_{\min }} q_{i, j}$

Hence, the OWFCR-CL model is the OWFCR model (2)-(14) with the addition of constraints (20)-(23), and the amended objective function (24).

\subsection{Example}

Figure 6 shows the solution to the OWFCR-CL problem for the considered HR3 test case. This optimal solution was found in 2931 seconds on a standard PC. If we assume not to have any limitation on branches, the company would select this structure only to cope with cable failures. Comparing with the optimized layout for the general OWFCR problem (in Figure 3), this layout is 3.9 M€ more expensive, including the cost for the redundant cables (in orange in the figure). Being able to quantify the extra cost for a loop structure is a very valuable input to the business case, making it possible to the company to evaluate alternative solutions (as batteries/diesel generators attached to each turbine). 


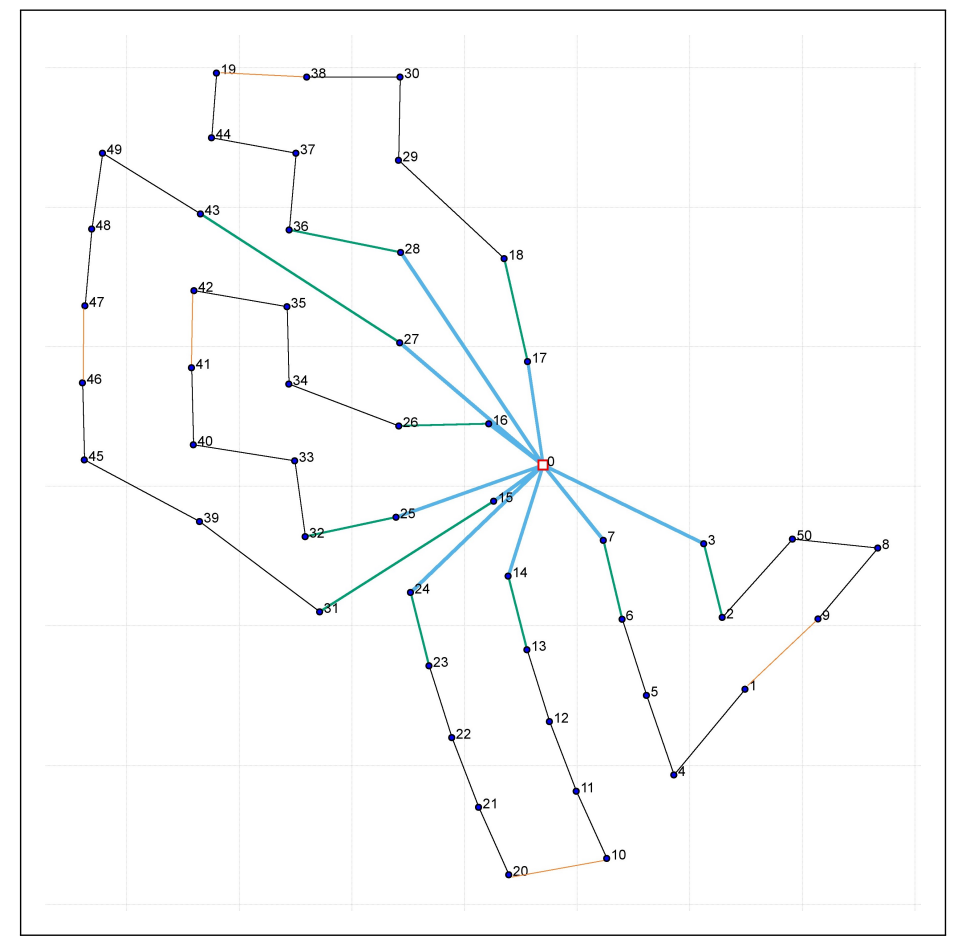

Fig. 6. Closed-loop structure (formulated as the OWFCR-CL problem); redundant cables are [1,9], [10,20], [19,38], [41,42] and [46,47] (in orange).

\section{Using OTMs instead of substations (OWFCR-OTM)}

The classical OWFCR problem assumes that substation(s) are fixed in advance. In very recent years, however, companies are questioning about the need for offshore substations, that are big and expensive structures involving a lot of components - while only the main transformer is required in practice. In 2015, Siemens [24] proposed an innovative structure, called Offshore Transformer Module (OTM), that is able to handle the transformer function through a smaller and cheaper hardware to be attached directly to the turbine foundations. The turbines with this OTM structure can be connected directly to shore, or to other OTM structures, through so-called export cables. Export cables differ from inter-array cables, in that they operate at a different voltage and have a much higher capacity (and a much larger price). Due to their different voltage, export cables cannot be connected directly to inter-array cables, but require the installation of a transformer-hence the need of the OTM. Figure 7 illustrates a typical cable routing involving export cables. 


\subsection{Mathematical formulation}

The first modification to our MILP model (2)-(13) consists in introducing a single "dummy substation" associated with a node $s$ located on shore, that represents the connection to the backbone electrical network. In addition, a special cable type $\tau$ is given on input, that corresponds to the export cable (with its capacity and unit cost). Also, we need to impose the following technical requirements: (a) no more than $\mu_{1}$ regular cables can enter a turbine, and (b) no more than $\mu_{2}$ export cables can enter each turbine. The above requirements can easily be modeled by the following additional constraints:

$$
\begin{aligned}
\sum_{i \in V: i \neq h} \sum_{t \in T: t \neq \tau} x_{i, h}^{t} \leq \mu_{1}, \quad h \in V_{T} \\
\sum_{i \in V: i \neq h} x_{i, h}^{\tau} \leq \mu_{2}, \quad h \in V_{T} .
\end{aligned}
$$

Finally, the fixed cost for each OTM $\left(c_{o t m}\right)$ can be added to the cost of each variable $x_{i, j}^{\tau}$, resulting in the new objective function:

(27) $\min \sum_{(i, j) \in A} \sum_{t \in T} c_{i, j}^{t} x_{i, j}^{t}+\sum_{(i, j) \in A} c_{o t m} x_{i, j}^{\tau}$.

Thus, the OWFCR-OTM model is the OWFCR model (2)-(14) with the addition of constraints (25)-(26), and the modified objective function (27). Note that our OWFCR-OTM formulation does not include the additional requirements of Sections 3 to 5 , as we only want to illustrate the potential of the new technology.

\subsection{Example}

We still use HR3 as an example of optimized solution considering OTMs. We estimated a price of $1 \mathrm{M} €$ for each OTM structure, and we set $\mu_{1}=2$ and $\mu_{2}$ $=1$. In this case the optimization considers also an extra cable (the export cable) that can support all turbines and has a cost of $1200 € / \mathrm{m}$. Figure 7 show the optimized layouts using the model presented in this Section. A "dummy substation" (node 0) was located on shore.

As opposed to the previous cases, that were solved to optimality in less than one hour, this test was run until the time limit of 1 hour, ending with a gap of $12 \%$.

We estimate the savings obtained by using OTMs instead of a substation in the following way: we fix the basic layout of Figure 3 and we add the cost for the export cable to shore (computed as $1200 € / \mathrm{m}$ ) and the substation cost of 


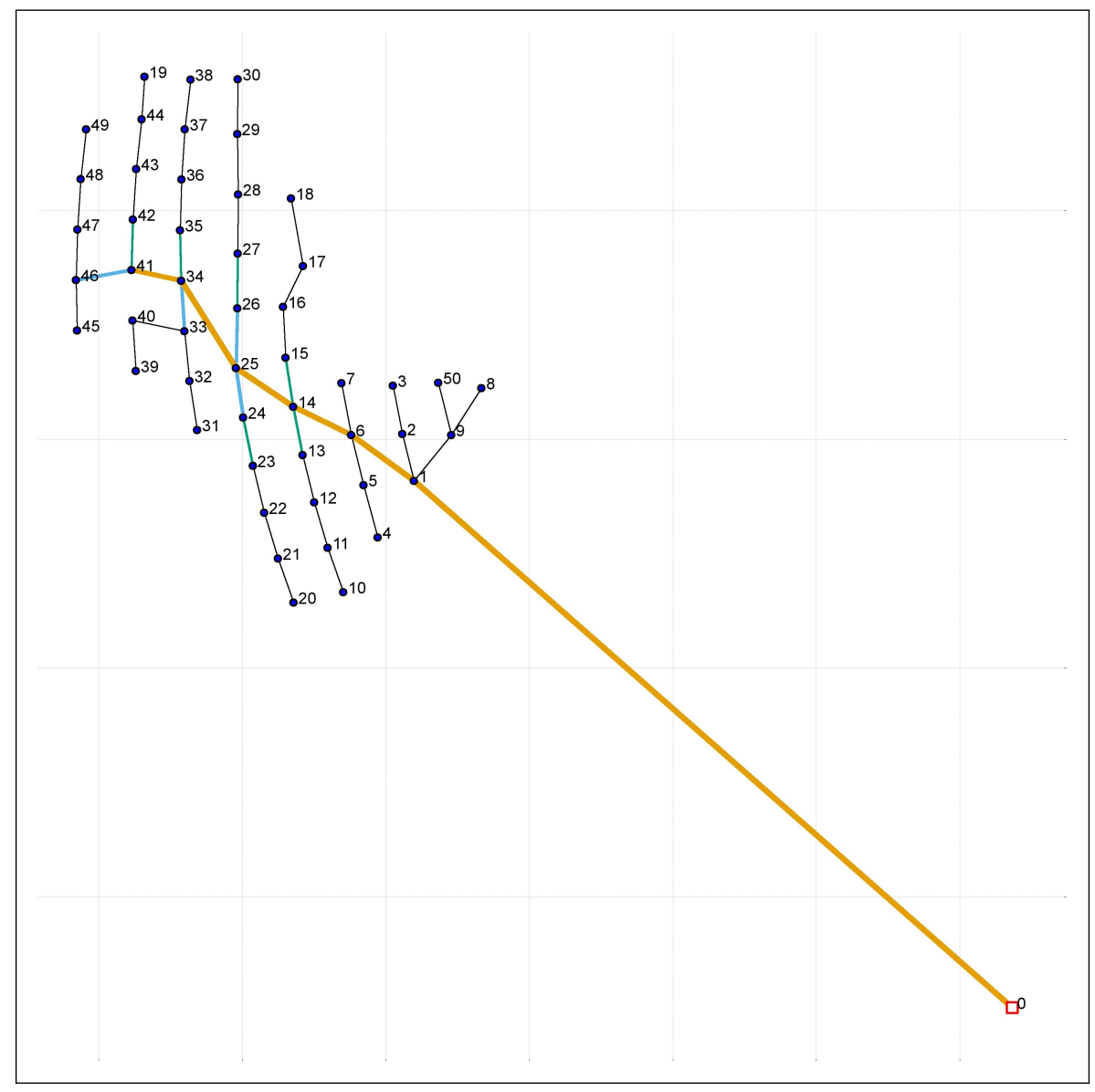

Fig. 7. Using OTMs allows for a saving of $101 \mathrm{M} €$.

$100 \mathrm{M} €$. The layout of Figure 7 then allows for a saving of $101 \mathrm{M} €$.

\section{A hybrid matheuristic/exact algorithm}

The use of ad-hoc heuristics to generate an initial solution has been proven to drastically the MILP solver, in particular for large size instances for which the exact solver is not able to deliver a satisfactory solution even if a large computing time is allowed. In our computational study, we used a matheuristic $[4,16,10]$ approach, which is a combination of mathematical programming with metaheuristics. The approach consists in designing sound heuristics on top of a black-box MILP solver, by just changing its input data in a way that favors finding a sequence of improved solutions. In our setting, the black-box MILP solver is an exact method applied to modified input. 
In our hybrid scheme [12], we first iteratively apply different refining schemes to the current best solution available. After repeating this matheuristics phase several times, we pass the best-available solution to the MILP solver and let it run to, possibly, solve the problem to proven optimality. Our matheuristic phase works as follows: at each iteration, we temporarily fix to 1 some $y$ variables according to a certain criterion (to be described later). Note that, by fixing to 1 some $y$ variables, we can automatically fix to 0 all the variables corresponding to crossing arcs, thus drastically reducing the size of the problem. We then apply the MILP solver to the corresponding restricted problem, and we warm start it by providing the current solution on input. We abort the execution as soon as a better solution is found, or a short time limit of a few seconds is reached. Then all fixed variables are unfixed, and the overall approach is repeated until a certain overall time limit (or a maximum number of trials) is reached. In order to quickly find feasible solutions, we use a relaxed version of the model in the matheuristic phase. This relaxed version allows for disconnected solutions, which are however strongly penalized. More specifically, we relaxed our MILP models with some additional (continuous) slack variables, representing the current loss at each disconnected turbine. These losses are minimized in the objective function: we used a very large (big-M) cost for these loss-variables, to ensure that the optimal solution will always have no disconnected turbines. For a more detailed description, we refer the reader to [12]. The approach proved to be very effective in the first iterations of our heuristic, when even finding a feasible (connected) solution can be problematic.

Different strategies can be used to decide which $y$ variables to fix to 1 . We used the following variable-fixing criteria. Let $y^{*}$ denote the best-available solution at the current iteration ( $y$ variables only).

Our first criterion simply selects with a certain probability (50\%) some arcs in the current best available solution. It fixes their corresponding variables to 1 in the new solution and re-optimizes only on the remaining arcs.

Our second criterion considers trees of turbines defined as follows. For each arc $(i, r)$ with $y_{i, r}^{*}=1$ that enters the substation, say $r$, we define the node set $S_{i}$ containing all the nodes that reach the substation $r$ passing through node $i$. In other words, $S_{i}$ contains all the predecessors of node $i$ in the antiarborescence corresponding to $y^{*}$. At each iteration we randomly select few trees to be optimized and we fix all the arcs not belonging to them.

Our third criterion partitions the wind farm into sectors of a certain angle from the substation. We then iteratively reoptimize each sector by fixing all the arcs outside the sector, i.e. all the arcs that involve two nodes not 
belonging to it.

The MILP models for the OWFCR problem variants were tested with our hybrid approach on the HR3 case. Most of these instances are solved to optimality within 1 hour. Table 1 reports the most important figures about our HR3 runs, including gap to optimality, number of branch-and-cut nodes, number of generated user cuts (no-cross constraints), and final computing time. In the right part of the table, we report also the statistics for the same instances run using the exact method only. We imposed a time limit of 1 hour to the MILP solver (IBM ILOG CPLEX 12.6) on the same standard $\mathrm{PC}$. The HR3 instance studied in our examples turned out to be an easy case, where both the exact and the hybrid approach performs similarly. According to Table 1, indeed, the matheuristic phase applied before the exact solver does not improve the final result for these easy instances. On the other hand, for hardest cases, it typically produces significantly better solutions within the time limit, improving the robustness (and hence the reliability) of our method. For a more detailed performance comparison between the exact and the matheuristic method, the reader is referred to [12].

Table 1

Computational information about the HR3 runs

\begin{tabular}{|c|c|c|c|c|c|c|c|c|c|c|c|c|}
\hline \multirow[b]{2}{*}{ Optimization model } & \multicolumn{6}{|c|}{ Matheruistic+exact algorithm } & \multicolumn{6}{|c|}{ Only exact algorithm } \\
\hline & $\begin{array}{c}\text { LP bound } \\
{[\mathrm{M} €]}\end{array}$ & $\begin{array}{c}\text { best sol } \\
{[\mathrm{M} €]}\end{array}$ & $\%$ gap & nodes & $\begin{array}{r}\text { no-cross } \\
\text { constr. }\end{array}$ & $\begin{array}{r}\text { final time } \\
(\mathrm{sec})\end{array}$ & $\begin{array}{c}\text { LP bound } \\
{[\mathrm{M} €]}\end{array}$ & $\begin{array}{c}\text { best sol } \\
{[\mathrm{M} €]}\end{array}$ & \%gap & nodes & $\begin{array}{r}\text { no-cross } \\
\text { constr. }\end{array}$ & $\begin{array}{r}\text { final time } \\
\text { (sec) }\end{array}$ \\
\hline OWFCR & 36.69 & 36.69 & 0 & 4964 & 137 & 176.41 & 36.69 & 36.69 & 0 & 4736 & 167 & 43.69 \\
\hline OWFCR-SS & 36.92 & 36.92 & 0 & 42796 & 199 & 598.33 & 36.92 & 36.92 & 0 & 55620 & 391 & 723.44 \\
\hline OWFCR-BP & 36.74 & 36.74 & 0 & 596 & 19 & 232.52 & 36.74 & 36.74 & 0 & 979 & 66 & 15.28 \\
\hline OWFCR-CL & 40.63 & 40.63 & 0 & 96649 & 972 & 2931.90 & 40.25 & 40.63 & 0.93 & 54499 & 1539 & 3600.00 \\
\hline OWFCR-OTM & 60.80 & 69.31 & 12.27 & 39450 & 2252 & 3600.00 & 60.82 & 69.31 & 12.24 & 57350 & 2974 & 3600.00 \\
\hline
\end{tabular}

\section{What-if analysis on real-world instances}

The MILP-based heuristic presented in Section 7 has been used on a set of real-world instances to test the economical impact of the new extensions of the model. The heuristic was programmed in C language on top of the commercial MILP solver IBM ILOG CPLEX 12.6, and was run on a standard PC with a 1-hour time limit.

\subsection{Test instances}

We tested our model on the real-world instances proposed in [12]. We considered five different real wind farms in operation in United Kingdom and Den- 
mark, and one new wind farm under construction. These parks are named Horns Rev 1, Ormonde, Dan Tysk, Thanet, and Horns Rev 3.

Our dataset includes old and new parks, with different power ratings and different number of turbines installed, and therefore represents a good benchmark for our tests. Each park has one substation with its own maximum number of connections (denoted by $C$ in our model).

Horns Rev 1 is one of the oldest large-scale wind parks in the world. It was built in 2002 in the North Sea, about $15 \mathrm{~km}$ from the Danish shore, and produces around 160 MW. Horns Rev 1 has 80 turbines Vestas 80-2 MW and $C=10$. Our second wind farm is Ormonde, located in United Kingdom, in the Irish Sea. It has a total capacity of $150 \mathrm{MW}$ (30 Senvion 5 MW turbines) and $C=4$. Our third park is Thanet, a bigger wind park with a capacity of 300 MW (100 Vestas 90-3 MW turbines) and $C=10$. When it was opened, in 2010, Thanet was the biggest offshore wind farm in the world. DanTysk offshore wind farm is located west of the island of Sylt and directly on the German-Danish border. With a total of 80 Siemens 3.6 MW turbines (288 MW), DanTysk can provide up to 400000 homes with green energy. It has $C=10$. Finally, the last layout refers to a preliminary layout for a new wind park, Horns Rev 3. Horns Rev 3 has a park capacity of $350 \mathrm{MW}$ and our preliminary layout uses 50 modern big-size turbines (Vestas $8 \mathrm{MW}$ ) and $C=12$. We already used this park as an illustrative example in the previous part of the paper. All the considered sites are owned by Vattenfall.

In these dataset we are also provided with different cable sets, indicated as cb01, cb02, cb03, cb04, cb05 and cb06.

Specific feasible combinations of site (i.e., wind farm) and cable set represent an instance in our testbed. Table 2 reports the main characteristics of instances, namely: the wind park layout (and its short name in parenthesis), the cable set name, the number of turbines in the layout, the number of cable types in the cable set, and the maximum number of connections to the substation $(C)$.

\subsection{What-if analysis}

The possibility of quickly evaluating the economical impact of alternative design choices is considered of fundamental importance by the Vattenfall's engineers, who make several "what-if" analyses before deciding the final cable routing to be implemented. We used our real-world dataset to analyze the impact of (i) branch vs string layout; (ii) branch vs loop structure; and (iii) 
Table 2

\begin{tabular}{|llrrr|}
\multicolumn{5}{c}{ Main characteristics of our test instances } \\
\hline park & cable set & n.turb. & n.cabl.types & $C$ \\
\hline Horns Rev 1 (wf01) & cb01 & 80 & 3 & 10 \\
& cb02 & 80 & 2 & 10 \\
Ormonde (wf03) & cb03 & 30 & 2 & 4 \\
& cb04 & 30 & 2 & 4 \\
Thanet (wf04) & cb01 & 80 & 3 & 10 \\
& cb04 & 80 & 2 & 10 \\
& cb05 & 80 & 2 & 10 \\
Dan Tysk (wf05) & cb04 & 100 & 2 & 10 \\
& cb05 & 100 & 2 & 10 \\
Horns Rev 3 (wf06) & cb03 & 50 & 2 & 12 \\
& cb04 & 50 & 2 & 12 \\
\hline
\end{tabular}

substation vs OTMs layouts.

\subsubsection{Economical value of layouts from OWFCR-SS vs OWFCR and OWFCR- $B P$}

As already discussed, our models were developed to help the engineers of the company to evaluate the impact of different decision choices in the design of the cable networks. Previously, engineers did not have any sound optimization tool to help them, so they were often designing cable routings by strings, as it was the easier case to handle manually. Our first task was therefore to compare the string structure with possible alternatives, using our MILP models. In particular, two different situations may occur: the selected turbine model for the park can handle multiple cable connections with no extra costs, or the selected turbine model can be connected to only one entering cable by default, and extra connections can be added at an additional price. We will therefore compare optimized string layouts (from our OWFCR-SS model) with layouts from the OWFCR model, for the first case, or with layouts from the OWFCR$\mathrm{BP}$ model in the latter case. In our first test we considered turbines that, by default, can be connected to at most 2 cables (one entering and one exiting), hence implementing a branch structure would imply extra costs. In this test we considered an extra cost of $25 \mathrm{k} €$ for having two cables entering a turbine $\left(\pi_{2}=\right.$ $25 \mathrm{k} €)$, and of $30 \mathrm{k} €$ for having three entering cables $\left(\pi_{3}=30 \mathrm{k} €\right)$; no extra costs was set for one entering cable, as this is the default setting $\left(\pi_{1}=0\right)$. The manual operator in this case would design the routing by strings, in order to have no extra costs. We therefore compared the cost of the optimized solutions 
considering explicit branch penalties in the model, with the string-structure optimized layouts. Table 3 reports the results: the first two columns identify the test instance (park and cable set), the third column reports the cost of the optimized solution considering branching penalties explicitly in the model, while the fourth column reports the cost of the optimized solution imposing a string structure (all costs in $\mathrm{M} €$ ). Finally, the last two columns report the difference branch-cost minus string-cost, hence negative values correspond to savings with respect to the string structure usually implemented by planners.

Table 3

Optimized branch vs string structure solutions (with branch extra-costs)

\begin{tabular}{|llrrrr|}
\hline park & cable set & OWFCR-BP sol. & OWFCR-SS sol. & diff & $\%$ diff \\
& & {$[\mathrm{M} €[$} & {$[\mathrm{M} \in]$} & {$[\mathrm{M} \in]$} & \\
\hline wf01 & cb01 & 19.45 & 19.45 & 0.00 & 0.0 \\
& cb02 & 22.62 & 22.62 & 0.00 & 0.0 \\
\hline \multirow{2}{*}{ w003 } & cb03 & 8.08 & 8.13 & -0.05 & -0.6 \\
& cb04 & 8.39 & 8.54 & -0.14 & -1.7 \\
\hline \multirow{2}{*}{ w04 } & cb01 & 39.06 & 39.10 & -0.04 & -0.1 \\
& cb04 & 38.77 & 39.64 & -0.87 & -2.2 \\
& cb05 & 49.54 & 49.54 & 0.00 & 0.0 \\
\hline \multirow{2}{*}{ w05 } & cb04 & 22.47 & 24.64 & -2.18 & -9.6 \\
& cb05 & 26.82 & 27.17 & -0.35 & -1.3 \\
\hline \multirow{2}{*}{ wf06 } & cb03 & 38.70 & 39.60 & -0.91 & -2.3 \\
& cb04 & 43.93 & 45.36 & -1.43 & -3.2 \\
\hline
\end{tabular}

It can be noticed that, having an optimization tool able to explicitly consider the branch costs in the optimization, results in large savings, compared to the classical approach of using only string structures. According to our experiments, the average saving using the branch-penalty model is of about $500 \mathrm{k} €$, with extreme cases with savings of more than $2 \mathrm{M} €$ (park wf05 with cable set cb04).

Some modern turbines are constructed to handle more than one entering cable, hence no extra costs for branches are paid and the OWFCR model of Section 2 can be used. In this case branches become even more attractive, as shown in Table 4 where we report a comparison between layouts from the OWFCR model and the OWFCR-SS one when there are no costs for branches. As in the previous table, the first two columns specify the instance, the next two report the cost of the optimized solution with the OWFCR model or the OWFCR-SS model (in M€), and the last tow columns give the difference. Our 
results confirm that, the classical planners' approach of connecting turbines by string is way more expensive than using a branch structure. The average savings when allowing for branches is of $600 \mathrm{k} €$, with extreme cases of savings over $2 \mathrm{M} €$. Due to the large number of possible configurations, it is not trivial to manually design an optimal layout, so a sound optimization tool is needed to achieve these savings.

Table 4

Branch vs string structure solutions (with no branch extra costs)

\begin{tabular}{|c|c|c|c|c|c|}
\hline park & cable set & $\begin{array}{r}\text { OWFCR sol. } \\
{[\mathrm{M} €]}\end{array}$ & $\begin{array}{r}\text { OWFCR-SS sol. } \\
{[\mathrm{M} €]}\end{array}$ & $\begin{array}{r}\text { diff } \\
{[\mathrm{M} €]}\end{array}$ & \%diff \\
\hline \multirow[t]{2}{*}{ wf01 } & cb01 & 19.44 & 19.45 & -0.02 & -0.07 \\
\hline & cb02 & 22.61 & 22.62 & -0.01 & -0.04 \\
\hline \multirow[t]{2}{*}{ wf03 } & cb03 & 8.05 & 8.13 & -0.08 & -0.9 \\
\hline & cb04 & 8.36 & 8.54 & -0.18 & -2.2 \\
\hline \multirow[t]{3}{*}{ wf04 } & cb01 & 38.98 & 39.10 & -0.12 & -0.3 \\
\hline & cb04 & 38.73 & 39.64 & -0.92 & -2.3 \\
\hline & cb05 & 49.35 & 49.54 & -0.19 & -0.4 \\
\hline \multirow[t]{2}{*}{ wf05 } & cb04 & 22.34 & 24.65 & -2.31 & -10.3 \\
\hline & cb05 & 26.64 & 27.17 & -0.53 & -1.9 \\
\hline \multirow[t]{2}{*}{ wf06 } & cb03 & 38.60 & 39.60 & -1.00 & -2.6 \\
\hline & cb04 & 43.73 & 45.36 & -1.62 & -3.7 \\
\hline
\end{tabular}

8.2.2 How to handle cable failures: using generators/batteries or closed loop? If the turbines are not equipped to survive disconnected from the electrical network, the company can apply different strategies to limit the damage in case of failures: either to buy external batteries or generators to be connected to each turbine, or to have a closed loop structure in the cable layout. Which of these two options is the most convenient one, it is not a trivial decision. Indeed, for a planner it is not easy to manually design the cheapest closed-loop structure and to exactly quantify how much is the extra investment incurred. Table 5 shows the results of this test using our OWFCR and OWFCR-CL models. According to our results, adopting a closed-loop structure can be up to $5 \mathrm{M} €$ more expensive and $3 \mathrm{M} €$ more expensive on average. Having these extra costs quantified can help the engineers making a data-driven decision. 
Table 5

OWFCR vs OWFCR-CL optimized solutions.

\begin{tabular}{|c|c|c|c|c|c|}
\hline park & cable set & $\begin{array}{r}\text { OWFCR sol. } \\
{[\mathrm{M} €]}\end{array}$ & $\begin{array}{r}\text { OWFCR-CL sol. } \\
{[\mathrm{M} €]}\end{array}$ & $\begin{array}{r}\text { diff } \\
{[\mathrm{M} €]}\end{array}$ & $\%$ diff \\
\hline \multirow[t]{2}{*}{ wf01 } & cb01 & 19.44 & 21.09 & -1.65 & -8.5 \\
\hline & cb02 & 22.61 & 24.55 & -1.94 & -8.6 \\
\hline \multirow[t]{2}{*}{ wf03 } & cb03 & 8.05 & 8.68 & -0.62 & -7.7 \\
\hline & cb04 & 8.36 & 9.17 & -0.82 & -9.8 \\
\hline \multirow[t]{3}{*}{ wf04 } & cb01 & 38.98 & 42.71 & -3.73 & -9.6 \\
\hline & $\mathrm{cb04}$ & 38.73 & 44.23 & -5.50 & -14.2 \\
\hline & $\mathrm{cb} 05$ & 49.35 & 54.62 & -5.27 & -10.7 \\
\hline \multirow[t]{2}{*}{ wf05 } & cb04 & 22.34 & 26.44 & -4.11 & -18.4 \\
\hline & cb05 & 26.64 & 29.77 & -3.13 & -11.8 \\
\hline \multirow[t]{2}{*}{ wf06 } & cb03 & 38.60 & 43.64 & -5.04 & -13.1 \\
\hline & cb04 & 43.73 & 48.90 & -5.17 & -11.8 \\
\hline
\end{tabular}

\subsubsection{Offshore Transformer Modules or substations?}

Finally, we tested the potential of the new OTM technology. To do so we considered a cost of $3 \mathrm{M} €$ for each OTM and of $1200 € / \mathrm{m}$ for the export cable. The position of the onshore connection point has been estimated for each wind park looking at [1]. The estimated cost of an offshore substation is $100 \mathrm{M} €$. In these tests we assumed that the company has to take care also of the export cable costs, and that the substation position has been fixed before running our model. In our test cases we used the real substation positions for each specific park, so we can reasonably assume that its location was optimized in the design phase. Therefore, the cost of the basic-model solution can be recomputed by adding the cost of the substation and the cost for the export cable. This is why the costs of the OWFCR solutions in Table 6 are higher than in the previous tables. Notice that, once the position of the substation is fixed, there is no room for optimizing the capital costs related to the export cable, that are therefore just computed in a post-processing phase. The OWFCROTM model of Section 6 was used to optimize the layout using OTMs instead of substations. Table 6 reports the comparison of the two technologies, and shows the potential of an optimized use of OTM technology. According to the table, savings can be as large as $67 \%$. The OTM optimized solution is, on average, $89 \mathrm{M} €$ cheaper than the classical one. 
Table 6

Cost of OWFCR layouts (including substation and export-cable costs) vs cost of using OTMs.

\begin{tabular}{|ccrrrr|}
\hline park & cable set & $\begin{array}{r}\text { OWFCR sol. } \\
{[\mathrm{M} \in]}\end{array}$ & $\begin{array}{r}\text { OWFCR-OTM sol. } \\
{[\mathrm{M} \in]}\end{array}$ & $\begin{array}{r}\text { diff } \\
{[\mathrm{M} €]}\end{array}$ & \%diff \\
\hline \multirow{2}{*}{ wf01 } & cb01 & 136.56 & 44.33 & 92.23 & 67.5 \\
& cb02 & 139.74 & 51.60 & 88.14 & 63.0 \\
\hline \multirow{2}{*}{ w03 } & cb03 & 149.12 & 54.98 & 94.13 & 63.1 \\
& cb04 & 149.42 & 55.17 & 94.25 & 63.0 \\
\hline \multirow{2}{*}{ w04 } & cb01 & 646.19 & 562.35 & 83.84 & 12.9 \\
& cb04 & 645.94 & 553.94 & 92.00 & 14.2 \\
& cb05 & 656.57 & 569.23 & 87.33 & 13.3 \\
\hline \multirow{2}{*}{ wf05 } & cb04 & 495.47 & 408.46 & 87.01 & 17.5 \\
& cb05 & 499.77 & 420.56 & 79.21 & 15.8 \\
\hline \multirow{2}{*}{ w06 } & cb03 & 172.82 & 81.71 & 91.11 & 52.7 \\
& cb04 & 177.95 & 83.69 & 94.26 & 52.9 \\
\hline
\end{tabular}

\section{Conclusions}

In the present paper we used Mixed Integer Linear Programming techniques to solve new versions of the classical offshore wind farm cable routing problem. Thanks to our close collaboration with Vattenfall BA Wind, we have been able to investigate the most recent trends on the market and to evaluate their impact on the cable routing.

Turbines are becoming more customized, allowing them to survive being disconnected from the grid in case of failures, or even to substitute substations through the so-called Offshore Transformer Modules (OTMs). Turbine customization opens up for new possibilities in the park layout, therefore it is crucial to have an optimization tool able to quickly evaluate the economical impact of new technologies on the wind park costs. In the present paper we have introduced a flexible and reliable optimization tool, that scales well for bigger parks and more complex constraints. We have been able to handle new features in the model (i.e., closed-loop structure, non-linear branch penalties and OTMs) and to quantify their effect on real-world instances. The outcome of our tests indicates that millions of euros are involved in these analyses, so decisions based on optimized solutions can lead to substantial savings for the company and, more generally, to cheaper transition toward sustainable energy. 


\section{Acknowledgement}

This work is supported by Innovation Fund Denmark. Thanks to Jesper Runge Kristoffersen, Iulian Vranceanu, Thomas Hjort, Kenneth Skaug, Urban Axelsson and Iver Slot from Vattenfall BA Wind who helped us in defining the cable routing extensions.

\section{References}

[1] 4cOffshore, website, http://www.4coffshore.com/offshorewind/ (2015), accessed: 2015-01-30.

[2] Bauer, J. and J. Lysgaard, The offshore wind farm array cable layout problem: a planar open vehicle routing problem, Journal of the Operational Research Society 66 (2015), pp. 360-368.

[3] Berzan, C., K. Veeramachaneni, J. McDermott and U. O. Reilly, Algorithms for cable network design on large-scale wind farms, Tech. Rep. Tufts University (2011).

[4] Boschetti, M., V. Maniezzo, M. Roffilli and A. R. Bolufé, Matheuristics: Optimization, Simulation and Control, in: Hybrid Metaheuristics, Springer Berlin Heidelberg, 2009 pp. 171-177.

[5] Carroll, P., B. Fortz, M. Labb and S. McGarraghy, A branch-and-cut algorithm for the ring spur assignment problem, Networks 61 (2013), pp. 89-103.

[6] Cerveira, A., A. D. Sousa, E. J. S. Pires and J. Baptista, Optimal cable design of wind farms: The infrastructure and losses cost minimization case, IEEE Transactions on Power Systems (2016), pp. 4319 - 4329.

[7] Dutta, S., "Data Mining and Graph Theory Focused Solutions to Smart Grid Challenges," Master's thesis, University of Illinois (2012).

[8] Dutta, S. and T. J. Overbye, A clustering based wind farm collector system cable layout design, in: Power and Energy Conference at Illinois (PECI), 2011, pp. 1-6.

[9] Fagerfjall, P., "Optimizing Wind Farm Layout - More Bang for the Buck Using Mixed Integer Linear Programming," Master's thesis, Department of Mathematical Sciences, Chalmers University of Technology and Gothenburg University, Goteborg, Sweden (2010).

[10] Fischetti, M. and M. Fischetti, "Matheuristics," Springer International Publishing, 2016 pp. 1-33. 
[11] Fischetti, M. and M. Monaci, Proximity search heuristics for wind farm optimal layout, Journal of Heuristics (2015), pp. 1381-1231.

[12] Fischetti, M. and D. Pisinger, Optimizing wind farm cable routing considering power losses, European Journal of Operational Research (2017).

[13] Fortz, B. and M. Labbé, Two-connected networks with rings of bounded cardinality, Computational Optimization and Applications 27 (2004), pp. 123148.

[14] Gonzlez, J. S., M. B. Payn, J. M. R. Santos and F. Gonzlez-Longatt, A review and recent developments in the optimal wind-turbine micro-siting problem, Renewable and Sustainable Energy Reviews (2014), pp. 133-144.

[15] Gonzlez-Longatt, F. M. and P. Wall, Optimal electric network design for a large offshore wind farm based on a modified genetic algorithm approach, IEEE Systems Journal (2012), pp. 164-172.

[16] Hansen, P., V. Maniezzo and S. Voß, Special issue on mathematical contributions to metaheuristics editorial, Journal of Heuristics 15 (2009), pp. 197-199.

[17] Hertz, A., O. Marcotte, A. Mdimagh, M. Carreau and F. Welt, Optimizing the design of a wind farm collection network, INFOR 50 (2012), pp. 95-104.

[18] Kis-orca, website, http://www.kis-orca.eu/downloads/ (2015), accessed: 2015-01-30.

[19] Labb, M., G. Laporte, I. R. Martn and J. J. S. Gonzlez, The ring star problem: Polyhedral analysis and exact algorithm, Networks 43 (2004), pp. 177-189.

[20] Li, D., C. He and Y. Fu, Optimization of internal electric connection system of large offshore wind farm with hybrid genetic and immune algorithm, in: Third International Conference on Electric Utility Deregulation and Restructuring and Power Technologies (DRPT2008), 2008, pp. 2476-2481.

[21] Pillai, A., J. Chick, L. Johanning and M. K. V. D. Laleu, Offshore wind farm electrical cable layout optimization, Engineering Optimization (2015), pp. 16891708.

[22] Qi, W., Y. Liang and Z.-J. M. Shen, Joint planning of energy storage and transmission for wind energy generation, Operations Research 63 (2015), pp. 1280-1293.

[23] Sannino, A., H. Breder and E. Nielse, Reliability of collection grids for large offshore wind parks, Proceedings of 9th International Conference on Probabilistic Methods Applied to Power Systems (2006). 
[24] Siemens, New AC Grid Access Solution from Siemens: Lighter, faster, cheaper, http://www. siemens.com/press/en/pressrelease/?press=/en/ pressrelease/2015/energymanagement/pr2015030151emen.htm.

[25] Zhao, M., Z. Chen and F. Blaabjerg, Optimisation of electrical system for offshore wind farms via genetic algorithm, IET Renewable Power Generation 3 (2009), pp. 205-216. 